\title{
Constitutional Trisomy 8 as First Mutation in Multistep Carcinogenesis: Clinical, Cytogenetic, and Molecular Data on Three Cases
}

\author{
Laura Seghezzi, Emanuela Maserati, Antonella Minelli, Claudia Dellavecchia, Paola Addis, Franco Locatelli, \\ Adriano Angioni, Pietro Balloni, Crescenzo Miano, Pietro Cavalli, Cesare Danesino, and Francesco Pasquali \\ Biologia Generale e Genetica Medica (L.S., E.M., A.M., C.De., P.A., C.Da., F.P.) and Clinica Pediatrica (F.L.), Università dì Pavia, Pavia; \\ Centro di Genetica Clinica, Università di Sassari, Sassari (E.M.); Servizio Immunotrasfusionale (A.A., P.B.) and Ematologia (C.M.), \\ Ospedale Pediatrico del Bambin Gesù, Roma; Servizio Trasfusionale, Ospedale di Cremona, Cremona (P.C.), Italy
}

Three patients, with constitutional trisomy 8 mosaicism (CT8M), who developed a malignancy are reported. The diagnoses were refractory anaemia, acute lymphoblastic leukaemia, and idiopathic myelofibrosis. In the child with acute leukaemia, the CT8M was diagnosed at birth due to severe dysmorphisms and malformations; the other two patients showed a milder phenotype, and the CT8M was diagnosed only after the finding of trisomy 8 in neoplastic cells. The review of eight similar, previously reported cases and the clinical, cytogenetic, and molecular studies performed in our patients led us to make the following observations: (1) CT8M predisposes to neoplasms, preferentially to myelo- or lymphoproliferative diseases; (2) a gene dosage effect for glutathione reductase in red blood cells was seen in two of our patients; (3) the wide phenotypic variation of CT8M was confirmed: trisomy 8 in neoplastic cells of phenotypically near-normal cases may be misinterpreted as acquired; and (4) molecular studies suggested a postzygotic origin of the trisomy in our three cases, with the supernumerary chromosome being of paternal origin in one case and of maternal origin in the other two. We postulate that the trisomy 8 in neoplasms may often occur by mitotic nondisjunction in an early embryonic multipotent cell and that what is usually interpreted as an acquired trisomy 8 may in fact be CT8M. The constitutional trisomy 8 would act as a pathogenetically important first mutation in multistep carcinogenesis. Whenever trisomy 8 is found in malignancies, the patient should be reevaluated clinically to exclude CT8M, and CT8M patients should be monitored for the possible development of malignancies. Genes Chromosom Concer 17:94-101 (1996). (c) 1996 Wiley-Liss, Inc.

\section{INTRODUCTION}

The phenotype of constitutional mosaicism for trisomy 8 (CT8M) usually includes mental retardation and dysmorphic features, but cases with a near-normal phenotype have also been reported (Chandley et al., 1980; Hasle et al., 1995). Acquired trisomy 8 is common in lympho- and myeloproliferative disorders (Mitelman, 1991). We review the few reported patients with CT8M who have developed a malignancy and report three cases of CT8M associated with haematological disorders.

\section{MATERIALS AND METHODS}

\section{Patients}

\section{Case I}

P.A. is a boy who was born in 1984 with a birth weight of $2,850 \mathrm{~g}$. At the age of 3 years, he presented with petechiae and was treated with intermittent courses of corticosteroids. In October 1991, he was found to be anaemic and thrombocytopenic. His blood indices were $8.9 \mathrm{~g} / \mathrm{dl}$ of haemoglobin ( $\mathrm{Hb}), 2.4 \times 10^{12} / 1$ of red blood cells (RBC), and 4.7 $\times 10^{9} / 1$ of white blood cells (WBC), with a normal differential count. Platelets were $11 \times 10^{9} / 1$, and megathrombocytes were present. The bone marrow was normocellular, with $2-3 \%$ blasts, normal myelopoietic series, very few megakaryocytes, and severe anomalies of the erythroid series that was hyperplastic with dyserythropoiesis, pycnotic nuclei, and cytoplasmic bridges. A diagnosis of refractory anaemia (RA) was made, and a chromosome analysis revealed CT8M (Table 1). The child's phenotype was examined for signs of this constitutional condition. A few dysmorphic changes were found, namely, macrocephaly, hypertelorism, broad nose, highly arched palate, short and wide neck, vertebral anomalies, hypoplastic nipples, bilateral syndactily of the second and third toes, hypoplastic nails, and mobile testes (Fig. 1a). The boy had slight mental retardation, with an IQ of 65 (normal values (n.v.) 75-100). Behaviour and speech problems were evident. In the following years, the haematological condition of the patient remained stable without need for therapy (June 1995).

\footnotetext{
Received February 7, 1996; accepted May 7, 1996.

Address reprint requests to Francesco Pasquali, C.P. 217, I-27100 Pavia, Italy.
} 
TABLE I. Results of Chromosome Analyses

\begin{tabular}{|c|c|c|c|}
\hline Case & Date & Material & Karyotype \\
\hline \multirow[t]{5}{*}{1} & $\begin{array}{l}22.10 .91 \\
04.12 .91\end{array}$ & $\begin{array}{l}\text { BM }^{2} \\
\text { PB-PHA } \\
\text { Fibroblasts }\end{array}$ & $\begin{array}{l}47, X Y,+8[18] / 46, X Y[1 I] \\
47, X Y,+8[23] / 46, X Y[27] \\
46, X Y[100]^{\mathrm{b}} \\
47, X Y,+8[33] / 46, X Y[37]^{c}\end{array}$ \\
\hline & & BM & $47, X Y,+8[19] / 46, X Y[3]$ \\
\hline & 14.04 .92 & BM & $47, X Y,+8[10] / 46, X Y[6]$ \\
\hline & 20.10 .92 & PB & $47, X Y,+8[5] / 46, X Y[5]$ \\
\hline & 15.06 .93 & PB & $47, X Y,+8[13] / 46, X Y[I I]$ \\
\hline \multirow[t]{3}{*}{2} & 02.06 .90 & PB-PHA & $47, X Y,+8[85] / 46, X Y[15]$ \\
\hline & 21.01 .91 & BM & $47, X Y,+8[5]^{d}$ \\
\hline & 29.09 .91 & Fibroblasts & $47, X Y,+8[36] / 46, X Y[14]^{e}$ \\
\hline \multirow[t]{3}{*}{3} & 22.01 .93 & PB-PHA & $47, X X,+8[13] / 46, X X[7]$ \\
\hline & 05.02 .93 & Fibroblasts & $47, X X,+8[18] / 46, X X[12]$ \\
\hline & $05.10 .93^{1}$ & & chi47, XX, $+8[3] / 46, X X[2] / 46, X Y[95]$ \\
\hline
\end{tabular}

aM, bone marrow direct preparations and 24-h cultures; PB-PHA, peripheral-blood PHA-stimulated cultures; PB, peripheral-blood unstimulated cultures. ${ }^{b}$ Cells from first growth halo.

cCells from second growth halo.

${ }^{\mathrm{F}} \mathrm{FSH}$ on BM nuclei with centromere-8-specific alphoid probe showed three signals in $90 \%$ of 100 cells.

eFISH on fibroblast nuclei showed three signals in $64 \%$ of 150 cells.

'After allogeneic bone marrow transplantation performed in August 1993.

\section{Case 2}

E.M. is a boy who was born in 1990 with a birth weight of 3,560 g. At birth, an echoscan showed enlargement of the left renal pelvis and calyces, with stenosis of the junction to the ureter, and right-sided hydronephrosis with renal hypoplasia. These findings were confirmed by urography. $\mathrm{X}$-ray examination showed diffuse condensing osteopathy, $1-\mathrm{cm}$ spondylolisis of the 11 th thoracic vertebra, short lower limbs, tubular bones with "bone-within-bone" appearance, iliac wing hypoplasia, and supernumerary ribs. The bone age was advanced (3 months). Electrocardiogram showed a slight biventricular hypertrophy. Reconstructive surgery permitted renal function. In January 1991, his liver and spleen were enlarged $(1 \mathrm{~cm}$ and $6 \mathrm{~cm}$ from costal margin, respectively), and blood indices were $8.3 \mathrm{~g} / \mathrm{dl}$ of $\mathrm{Hb}, 3.47 \times 10^{12} / 1$ of RBC, $15 \times 10^{9} / 1$ of $\mathrm{WBC}$, and $75 \times 10^{9} / 1$ of platelets. The bone marrow was almost monomorphic, with 94\% L1-type blasts with a common immunologic phenotype (CD24 89\%, CD9 75\%, CD10 $84 \%$, OK DR 82\%, CD38 68\%, Cy Ig neg, CD20 6\%). Acute lymphoblastic leukaemia (ALL) was diagnosed. A marrow examination 1 month after the beginning of polychemotherapy showed normal morphology and disappearance of blasts.

The child was reevaluated in April 1992 (Fig. $1 \mathrm{~b}, \mathrm{c})$. He was now hypotonic, did not walk, and psychomotor delay was evident. The skin was pale with cutis marmorata. Craniofacial dysmorphisms included microcephaly ( $<3$ rd percentile), hypertelorism, micrognathia, everted and thick lower lip, large nose, horizontal dimple on the chin, and lowset ears. We observed a short and wide neck, narrow shoulders, long trunk, kyphoscoliosis with dorsal boss, funnel chest with pectus excavatum, short hands with deep palmar furrows and nail dysplasia, genu valgum and pes valgus, camptodactily of the 2nd left toe, very deep plantar furrows, and hypospadia. The liver and spleen were still slightly enlarged.

\section{Case 3}

V.R. is a woman who was born in 1964. Although she presented with slight mental retardation, she attended and finished primary school. Since the age of 12 years, she has had regular menses. In January 1989, a macrocytic anaemia (Hb 9.7 g/dl) was found, and treatment with vitamins was ineffective. In December 1992, the anaemia worsened, and her blood indices showed $5.6 \mathrm{~g} / \mathrm{dl}$ of $\mathrm{Hb}, 6 \times$ $10^{9} / 1$ of WBC with $33 \%$ lymphocytes, $52 \%$ neutrophils, and $15 \%$ blasts. The blasts (alkaline phosphatase and CD34 positive) were large, with a high nucleus/cytoplasm ratio. The cytoplasm was basophilic, with fine azurophilic granules, and anisopoikilocytosis was present. The bone marrow was hy- 

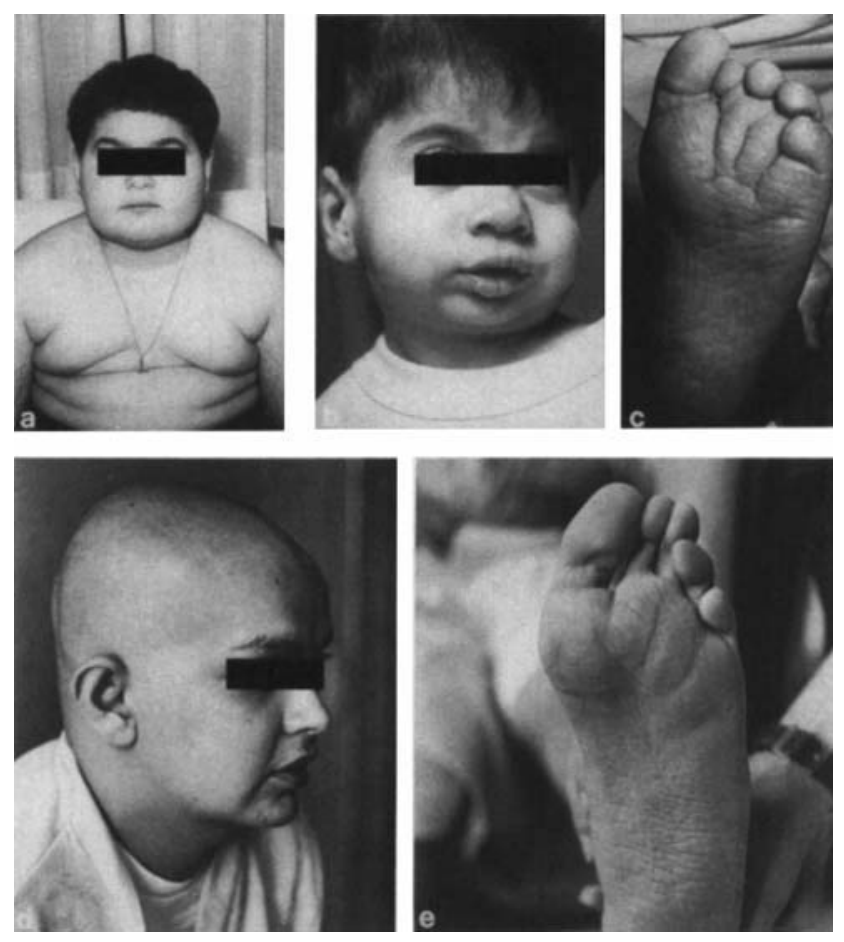

Figure I. a: Phenotype of case I, aged 7 years. b,c: Face and left foot of case 2, aged 2 years. d,e: Face and left foot of case 3 , aged 28 years.

percellular (85-90\%), with hyperplasia of the granulocytic and megakaryocytic series. The granulocytic series showed various steps of development; the megakaryocytes were in clusters and dystrophic. The number of erythroid cells was decreased, and only normoblasts and proerythroblasts were observed. Stromal cells were diffusely abundant, with some lymphoid reactive clusters. A diagnosis of idiopathic myelofibrosis was made, and she was treated only with transfusions. Attempts to obtain cytogenetic results on bone marrow samples failed, but the karyotype from stimulated blood cultures revealed a CT8M. The phenotype was reevaluated in view of the constitutional anomaly and showed a few dysmorphic changes: moderate palpebral ptosis and strabismus, horizontal dimple on the chin, everted lower lip, highly arched palate, wide neck, narrow shoulders and long trunk, dorsolumbar kyphoscoliosis, one supernumerary nipple, clinodactily of the 5 th finger, hallux valgus, sandal gap of toes, hammer toes, and deep palmar and plantar furrows (Fig. 1d,e). Gastric hypotonia with slow peristalsis and ptosis of the transverse colon was found, but without organic lesions. The IQ was 83 (n.v. 90-100). At the end of August 1993, she received a bone marrow transplant from her HLA-identical brother. She died of interstitial pneumonia in March 1994.

\section{Cytogenetic Methods}

Patients 1 and 3 were first investigated at the moment of diagnosis of their haematological disorders on bone marrow and on stimulated peripheral blood cultures. In patient 2, a stimulated blood culture was performed at birth, and a chromosome analysis was repeated on bone marrow when ALL was diagnosed. In addition, CT 8M was checked on fibroblast cultures from skin biopsies from all of the patients. Clones were established from fibroblasts and used for molecular studies only after at least 50 mitoses showed a nonmosaic karyotype.

Chromosome analyses were performed according to standard techniques, and the karyotypes for all of the patients were analyzed by QFQ and GTG banding.

\section{Biochemical Methods}

Glutathione reductase (GSR), which maps to 8p12 (Oshima et al., 1994), was assayed in RBC from patients 1 and 2 and from their parents by the method of George and Francke (1976), with minor modifications. The results of enzyme determinations are expressed as ratios between the activities of propositi and the mean of their parents' activities (Rts) to minimize the relevance of individual variations. 


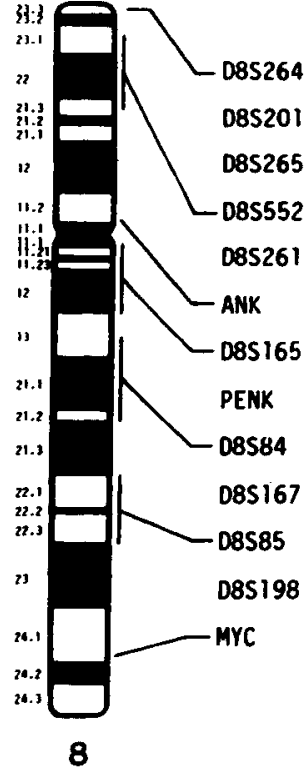

Figure 2. Results of STR analyses in the three patients and their parents. Loci tested are listed after their map order; exact localization is shown only for some of them. The results obtained for each locus are entered with letters, the letter a being the allele with the lowest molecular weight. Columns headed by the number 46 refer to cloned

The results were compared with a group of controls and with a group of patients with constitutional duplication of the region of chromosome 8 which includes the gene for GSR.

Peptidase A (PEPA) and malate dehydrogenase (MDH1), whose genes are mapped on chromosomes 18 and 2, respectively, were tested as control enzymes.

\section{Molecular Methods}

To determine the origin of the extra chromosome 8 , we performed a molecular analysis of 13 short tandem repeats (STR) scattered along chromosome 8 on DNA from the 46 and 47 cloned fibroblasts of the three patients and from peripheral WBC of their parents. In addition, WBC were tested in patients 1 and 3 . The STR were chosen among those with high heterozygosity and are listed in Figure 2. Methods for polymerase chain reaction (PCR) amplification and product detection were described elsewhere (Floridia et al., 1996).

\section{RESULTS}

\section{Cytogenetic Results}

The results are listed in Table 1 . In case 1 , the trisomic line in fibroblasts was observed only on a
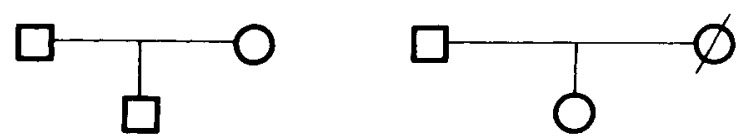

46,47
$b c, \quad b c, b b c, \quad a b$
$b c, a c, a c, \quad a d$
$a b, b b, b b, \quad b c$
$b d, \quad b c, b c, \quad a c$
$a d, a c, a c c, \quad b c$
$a c, a b, a b b, \quad a b$
$a a, a b, a b b, \quad a b$
$b b, \quad b b, b b, \quad a b$
$a c, \quad b c, b c, \quad b d$
$a c, \quad b c, b c, \quad b d$
$a b, \quad a d, a d, \quad a d$
$b c, a b, a b, \quad a c$
$c c, \quad b c, b c, \quad a b$

Case 2

$\overline{46} \mathbf{4 7} \overline{\mathbf{P B}}$
$b b, a b, a a b, a a b$
$a b, a c, a c, a c$
$a a, a a, a d, a d$
$a b, a b, a a b, a a b$
$a a, a a, a a, a d$
$a b, a b, a b b, a b$
$a a, a b, a b b, a b b$
$a b, a b, a b, a b$
$a a, a d, a a, a d$
$a a, a b, a b, a b$
$b b, a b, a a b, a a b$
$b c, a c, a a c, a a c$
$a b, a b, a b, a b$

Case 3 diploid fibroblasts, those by the number 47 to cloned trisomic 8 fibroblasts, and PB indicates analysis on WBC. Evidence of duplication of one allele is indicated by three letters. Results informative for parental origin of the supernumerary chromosome are in boldface.

second chromosome preparation made on the second growth halo from skin fragments. In case 2 , the presence of trisomy 8 in bone marrow and skin fibroblasts was also demonstrated with interphase cytogenetics (FISH with a centromere-8-specific alphoid probe).

\section{Gene Dosage Studies}

The results of the assays for GSR, PEPA, and MDH1 are listed in Table 2. In the control group, the Rts were found very close to the expected ones for all enzymes.

The patients with constitutional duplication of at least $8 \mathrm{p} 12$ showed increased Rts for GSR when compared with controls $(\mathrm{t}=8.34, p<0.001)$, corresponding to a gene dosage effect.

In patients 1 and 2, the Rts for the control enzymes was normal, whereas the one for GSR was increased (Table 2). In case 1, the Rts for GSR was intermediate between controls ( 2 alleles) and patients with $8 \mathrm{p}$ duplications ( 3 alleles). The value found could be obtained if $50 \%$ of the RBC were derived from cells with three alleles, which is in agreement with the mosaic status of the patient in the various tissues studied (Table 1). 
TABLE 2. Results of Gene Dosage Studies ${ }^{a}$

\begin{tabular}{cccc}
\hline & GSR & PEPA & MDHI \\
\hline $\begin{array}{c}\text { Controls } \\
\text { (n) }\end{array}$ & $1.07 \pm 0.14$ & $0.92 \pm 0.19$ & $0.99 \pm 0.11$ \\
$\begin{array}{c}(16) \\
\text { dup(8p) }\end{array}$ & $1.76 \pm 0.29$ & $(11)$ & $(10)$ \\
(n) & $(12)$ & - & - \\
Case 1 & 1.45 & 0.86 & - \\
Case 2 & 2.34 & - & 1.15 \\
\hline
\end{tabular}

'The figures refer to ratio between the enzymatic activity of the child and the mean of the activities of his parents (Rts). The expected Rts for normal subjects is $I$.

In case 2, the Rts was similar to the highest values found in $\operatorname{dup}(8 p)$ patients (Table 2 ), which agrees with the higher percentage of trisomic cells observed ('Table 1)

Case 3 was not tested because of the frequent blood transfusions.

\section{Molecular Results}

All the results are entered in Figure 2 and partially shown in Figure 3. When comparing allelic data on the cloned fibroblasts for each patient, we found the same alleles in cells with and without trisomy 8 . We never observed three different alleles in the 47 cell line. At least three of the STR showed a clear difference in the intensity of the two alleles in the trisomic cells of each of the patients (Fig. 3), which we interpret as evidence of duplication of this allele (Fig. 2). The alleles showing increased intensity were consistently paternal in origin in case 1 and maternal in origin in cases 2 and 3 (Figs. 2, 3). Analysis of additional control autosomal STR (D4S2361, D6S1003, D15S822, D13S782) demonstrated the presence of the expected paternal or maternal (cases 1 and 2) and paternal (case 3) alleles (data not shown).

\section{DISCUSSION}

The risk of neoplasms in patients with constitutional chromosome anomalies is well known for trisomy 21 (Sandberg, 1991) and was discussed for other anomalies by Alimena et al. (1985) and Hecht (1987), who, however, did not study any CT8M case. We found eight reports of single cases of patients with CT $8 \mathrm{M}$ who developed a neoplasm, and these are listed in Table 3 with the three present cases.

Does CT8M predispose to neoplasms and, in particular, to myelo- or lymphoproliferative disorders? Cancer is the result of the accumulation of different genetic lesions as activation of proto-on- cogenes, inactivation of tumor suppressor genes, or chromosome abnormalities. The molecular significance of many of these abnormalities is not known, but they are very frequent in some tumor types (Solomon et al., 1991; Heim, 1992), as is the case with trisomy 8 . The fact that trisomy 8 cells are present in various tissues in CT8M, e.g., in the bone marrow, could predispose to malignant transformation. This action is similar to that of retinal cells bearing a heritable mutation of the $R B 1$ gene, which makes them prone to develop retinoblastoma (Knudson, 1971).

The exact prevalence of CT8M is not known but is certainly low. Procter et al. (1984) stated that fewer than 100 cases had been reported. Nielsen and Wohlert (1991) found 1 case out of 34,910 newborns; we found 2 out of 40,140 routine chromosome analyses; Hecht and Hecht (1987) mentioned that no case had been found in 64,887 liveborns; and Hasle et al. (1995) estimated a prevalence of 1 in 25,000 . In comparison with these numbers, the fact that we are aware of 11 cases of CT8M who developed a malignancy (Table 3 ) cannot be a coincidence. Therefore, we postulate that CT $8 \mathrm{M}$ predisposes to neoplasms.

The study of GSR activity in patients 1 and 2 demonstrated, as expected, a gene dosage effect (Table 2). An increased activity of GSR was already reported in acquired trisomy 8 by de la Chapelle et al. (1978) and Sadamori et al. (1983). Three relevant points should be mentioned. First, trisomy 8 is detectable indirectly through gene dosage studies in cells such as RBC, where chromosome analyses cannot be made. This fact may help studies in lineage involvement. Second, it is possible to correlate the level of activity to the relative proportions of disomic and trisomic cells (Tables 1, 2). Third, the effect of an acquired trisomy on the neoplastic cell phenotype is mediated by increased dosage of gene products whose loci are on the involved chromosome. Therefore, gene dosage studies may explain some features of the neoplastic cell, as was suggested for monosomy 7 (Minelli et al., 1990).

A peculiar observation was made during fibroblast analysis of patient 1 . The karyotype was normal in 100 mitoses from the first growth halo from skin fragments, whereas the second growth from the same fragments showed 33 trisomic cells out of 70 analysed (Table 1 ). We have no explanation for this unexpected finding.

The wide variation of phenotypes in CT8M has been discussed (Chandley et al., 1980; Camurri and Chiesi, 1991; Kapaun et al., 1993; McDonald- 
Case 1

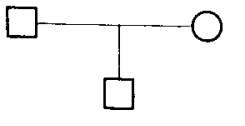

$\overline{4647 \mathrm{~PB}}$

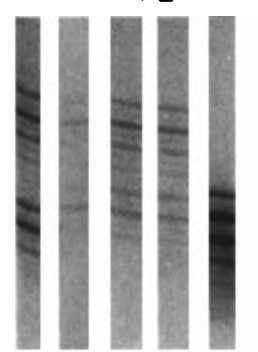

D8S84

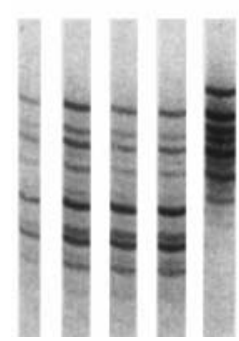

085552
Case 2

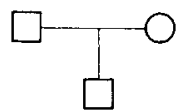

$\overline{46 \overline{47}}$

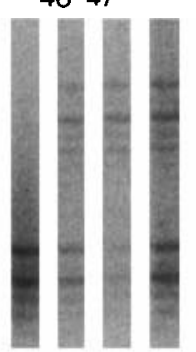

D8S 165

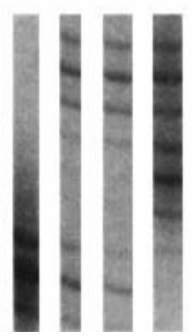

D8S261
Case 3
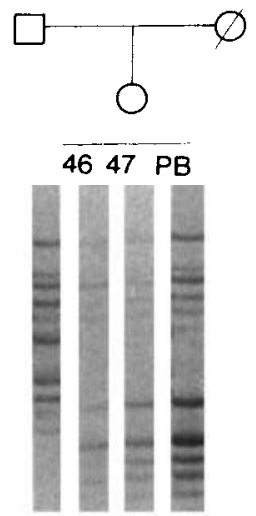

D8S198

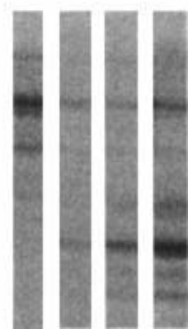

D8S85
Figure 3. Examples of results obtained in STR analysis of the three patients and their parents. Headings of lanes as in Figure 2 . In case 1 , the intensity of the paternal allele appears stronger than the maternal one

McGinn et al., 1993; Hasle et al., 1995; Mark et al., 1995). Schinzel (1994) listed 46 signs found in at least $10 \%$ and 19 found in at least $20 \%$ of 102 cases of CT8M. Some of these signs were seen also in our patients; case 2 presented the most signs and he was the only one with CT8M diagnosed before the neoplastic disorder. Only three manifestations were common to our three patients, namely mental retardation, short neck, and scoliosis. Dysmorphisms such as narrow shoulders, hypoplastic nails, hallux valgus, and supernumerary nipples, which may be expected and were present in our cases, are easily overlooked. Thus, the phenotype of CT8M can escape recognition, as happened in our cases 1 and 3 , because the manifestations may be minor. Even mental retardation is present in only $71 \%$ of cases (Schinzel, 1994).

Haas and Seyger (1993) postulated that some of the trisomies of neoplasms may originate during meiosis, leading to a constitutional mosaic with the trisomic line limited to neoplastic cells. We knew from fibroblast analyses that the trisomy in our cases was constitutional, and we tried to demon- for both loci in the 47 cells and in PB. A similar difference of intensity of the two alleles in 47 and PB cells is shown for both loci of cases 2 and 3 , in whom the maternal allele is stronger.

strate the meiotic origin of the extra chromosome 8 . When analysing molecular polymorphisms with this aim, the trisomic cell line is expected to show both alleles of the heterozygous parent contributing the supernumerary chromosome (nonreduction to homozygosity) or only one of them (reduction to homozygosity) in various combinations for the different loci, depending on a meiosis 1 or meiosis 2 nondisjunction and on possible crossing-over events (Antonarakis et al., 1993). If a meiotic error occurred, then the 46 cell line would originate by loss of the supernumerary chromosome, resulting in uniparental disomy in one-third of cases.

We never observed three different alleles at any of the loci analysed (Fig. 2). In 13 instances, allele distribution of cases 1 and 2 excluded the presence of two different alleles contributed by either parent. In case 3 , where only paternal DNA was available, we never observed evidence of two different nonpaternal alleles, and two loci showed only one of the two paternal alleles. Paternal and maternal contributions were present in the 46 cell lines of the three patients. 
TABLE 3. CT8M Cases Associated With Neoplasms

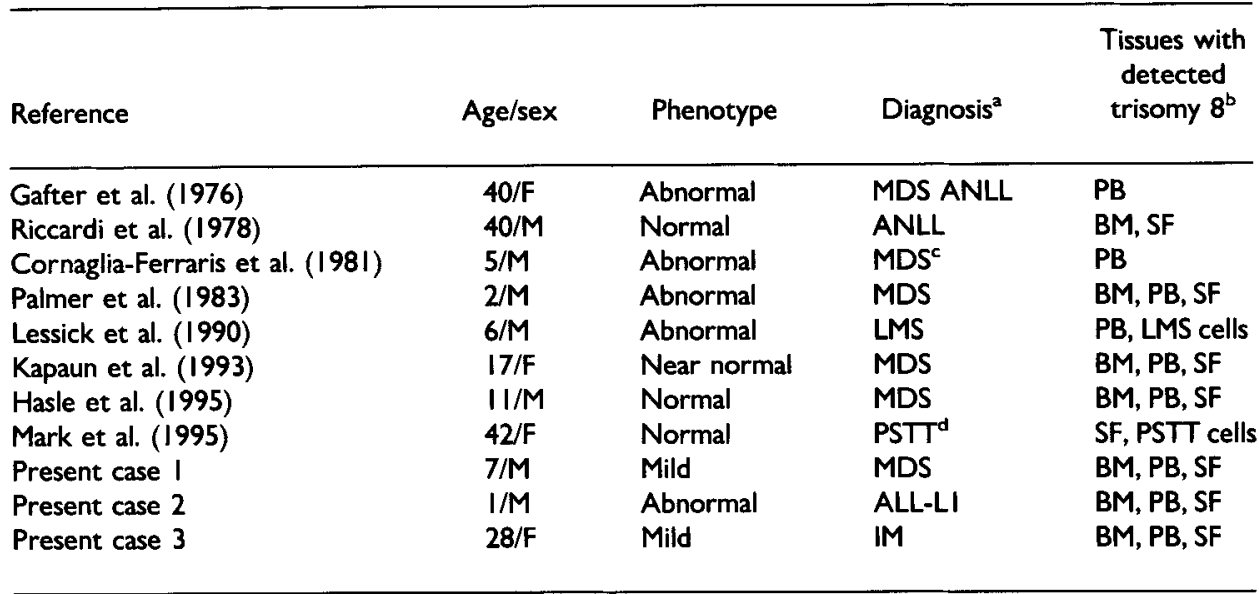

aMDS, myelodysplastic syndrome (includes disorders such as "aplastic anaemia followed by acute leukaemia", Gafter et al., 1976, "juvenile chronic granulocytic leukaemia", Palmer et al., 1983, "atypical Ph-negative chronic myelogenous leukaemia", Kapaun et al., 1993, and "refractory anaemia with excess of blasts", Hasle et al., 1995); ANLL, acute nonlymphocytic leukaemia; LMS, leiomyosarcoma; PSTT, placental site trophoblastic tumor; ALL-LI, acute lymphoblastic leukaemia of $L I$-type FAB; IM, idiopathic myelofibrosis.

'PB, peripheral blood; BM, bone marrow; SF, skin fibroblasts.

CMDS is not in fact diagnosed in this case but only suspected on the basis of in vitro colony-forming capacity of BM cells.

'The interpretation of this case is controversial. CT8M is present in this woman and demonstrated by SF chromosome analysis, whereas PSTT is a tumor originating in trophoblastic cells, that is, cells of embryonic origin; trisomy 8 , however, is also present in these tumor cells.

Thus, we failed to obtain any evidence of a meiotic error, and the best explanation of our data is a mitotic postzygotic nondisjunction.

Evidence for paternal or maternal origin of the supernumerary chromosome 8 was inferred by the uneven intensity of the alleles in the trisomic clones of the three patients (Figs. 2,3). No difference in intensity was observed in the 46 clones. In peripheral blood, where a mixture of 46 and 47 cells was present and at least $50 \%$ of the metaphases showed trisomy 8 (Table 1), we observed results similar to those of cloned 47 fibroblasts, with the exception of a single marker (ANK) in case 3 (Fig. 2).

We are well aware of the caution that must be used in quantitative interpretation of PCR results. However, because the more intense band could be consistently referred to the father in case 1 and to the mother in cases 2 and 3, we interpreted these findings as evidence of duplication of paternal and maternal alleles, respectively. Robinson et al. (1995) identified a paternal origin in two other cases of CT8M with the same method. Therefore, a random occurrence of parental origin seems to be present in the five cases of CT8M studied so far, as in the postzygotic trisomy 21 mosaics studied by Antonarakis et al. (1993).

We differ somewhat with the hypothesis by Haas and Seyger (1993) in that the origin of trisomy 8 in neoplasms is not necessarily meiotic but may often be mitotic-postzygotic. The point is that, whatever the origin, what is usually thought to be acquired trisomy 8 may in fact be a CT8M. This may arise from a postzygotic mitotic nondisjunction in an early embryonic multipotent cell, from which different tissues may originate, instead of a cell of an already differentiated tissue, as would be true of bone marrow when trisomy 8 is associated with myeloproliferative disorders. Thus, the neoplastic risk of CT8M would be explained by the constitutional presence of a primary chromosome mutation.

Our results support this hypothesis because we observed trisomy 8 in different cell types of our patients, such as fibroblasts, WBC, and RBC (as deduced by gene dosage of GSR), and our molecular analysis fits with a postzygotic origin of the CT8M.

The presence of trisomy 8 in nonmyeloid cells in a case of acute myeloid leukaemia was reported by El-Rifai et al. (1996), who also found it in lymphocytes and discussed its relevance in the development of leukaemia. No further data are provided to reveal whether this case might in fact be a CT8M.

It is relevant that 9 of 11 cases of CT8M listed in Table 3 developed haematologic disorders in which a trisomy 8 is frequent. Of the two solid tumors, one is a leiomyosarcoma, a neoplasm in which trisomy 8 has been found as an acquired 
anomaly, although not among the most frequent ones (Sandberg and Bridge, 1994). No consistent cytogenetic data are available for PSTT (Table 3).

The variable phenotype of CT8M may be responsible for the failure of recognition in near-normal or mild cases; when such cases in turn develop a neoplasm, the trisomy 8 in neoplastic cells is thought to be acquired. We stress the fact that 6 of the 11 cases listed in Table 3 were found to be CT8M only after the demonstration of a trisomy 8 in neoplastic cells.

In conclusion, we suggest that, when a trisomy 8 is found in neoplastic cells, the patient should be reevaluated clinically to check for the possibility of a CT8M, and, conversely, that when a CT8M is diagnosed the patient should be accurately observed for the risk of neoplasms and, in particular, of haematological neoplasms.

\section{ACKNOWLEDGMENTS}

We thank Professor M. Fraccaro for his help in the preparation of this paper. The study was supported by grants from CNR, PF-ACRO (93.02211.39, 94.01178.39, 95.00409.39), from Associazione Italiana per la Ricerca sul Cancro (AIRC), Milano, and from Regione Lombardia, Ricerche finalizzate in campo sanitario. The financial support of Telethon to Project C.13 is also acknowledged.

\section{REFERENCES}

Alimena G, Billström R, Casalone R, Gallo E, Mitelman F, Pasquali $\mathrm{F}$ (1985) Cytogenetic pattern in leukemic cells of paticnts with constitutional chromosome anomalies. Cancer Genet Cytogenet 16:207-218.

Antonarakis SE, Avramopoulos D, Blouin J, Conover Talbot C Jr, Schinzel AA (1993) Mitotic errors in somatic cells cause trisomy 21 in about $4.5 \%$ of cases and are not associated with advanced maternal age. Nat Genet 3:146-150.

Camurri L, Chiesi A (1991) A three-year follow-up on a child with low level trisomy 8 mosaicism which was diagnosed prenatally. Prenat Diagn 11:59-62.

Chandley AC, Hargreave TB, Fletcher JM, Soos M, Axworthy D, Price WH (1980) Trisomy 8. Report of a mosaic human male with near-normal phenotype and normal $I Q$, ascertained through infertility. Hum Genet 55:31-38.

Cornaglia-Ferraris P, Ghio R, Barabino A, Perlino GF, Maggio A, Parodi MT, Massimo L (1981) Diminished in vitro colony forming capacity of bone marrow cells in a case of chromosome 8 trisomy (mosaicism): Criteria for "high risk" pre-leukemia syndrome. Boll Ist Sieroter Milan 60:69-73.

de la Chapelle A, Vuopio P, Icén A (1978) Trisomy 8 in the bone marrow associated with high red cell glutathione reductase activity. Blood 47:815-826.

El-Rifai W, Larramendy ML, Ruutu T, Knuutila S (1996) Lymphoid involvement in a patient with acute myeloid leukemia: A direct phenotypic and genotypic study of single cells. Genes Chromosom Cancer 15:34-37.

Floridia G, Piantanida M, Minelli A, Dellavecchia C, Bonaglia C, Rossi E, Gimelli G, Croci G, Franchi F, Gilgenkrantz S, Grammatico P, Dalprà L, Wood S, Danesino C, Zuffardi O (1996) The same molecular mechanism at the maternal meiosis I produces mono- and dicentric 8p duplications. Am J Hum Genet 58:785796.
Gafter U, Shabtai F, Kahn Y, Halbrecht I, Djaldetti M (1976) Aplastic anemia followed by leukemia in congenital trisomy 8 mosaicism. Ultrastructural studies of polymorphonuclear cells in peripheral blood. Clin Genet 9:134-142.

George DL, Francke U (1976) Gene dosage effect: Regional mapping of human glutathione reductase on chromosome 8. Cytogenet Cell Genet 17:282-286.

Haas OA, Seyger M (1993) Meiotic origin of trisomic neoplasms. Cancer Genet Cytogenet 70:112-116.

Hasle H, Clausen N, Pedersen B, Bendix-Hansen K (1995) Myelodysplastic syndrome in a child with constitutional trisomy 8 mosaicism and normal phenotype. Cancer Genet Cytogenet 79: $79-81$.

Hecht F (1987) Risk of hematologic malignancy with constitutional chromosome abnormalities. Cancer Genet Cytogenet 24:375377.

Hecht F, Hecht BK (1987) Aneuploidy in humans: Dimensions, demography, and dangers of abnormal numbers of chromosomes. In Vig BK, Sandberg AA (eds): Aneuploidy. Part A: Incidence and Etiology. New York: Alan R. Liss, pp 9-49.

Heim $S$ (1992) Is cancer cytogenetics reducible to the molecular genetics of cancer cells? Genes Chromosom Cancer 5:188-196.

Kapaun P, Kabisch H, Held KR, Walter TA, Hegewisch S, Zander AR (1993) Atypical chronic myelogenous leukemia in a patien with trisomy 8 mosaicism syndrome. Ann Hematol 66:57-58.

Knudson AG (1971) Mutation and cancer: Statistical study of retinoblastoma. PNAS 68:820-823.

Lessick M, Israel J, Szego K, Wong P (1990) Leiomyosarcoma in a patient with trisomy 8 mosaicism. J Med Genet 27:643-644.

Mark HFL, Ahearn J, Lathrop JC (1995) Constitutional trisomy 8 mosaicism and gestational trophoblastic disease. Cancer Genet Cytogenet 80:150-154.

McDonald-McGinn DM, Grace K, Spinner NB, Emanuel B, Zackai EH (1993) Clinical and cytogenetic variability in trisomy 8 mosaicism. Am J Hum Genet 53 (suppl): 222.

Minelli A, Piantanida M, Maserati E, Campagnoli E, Pasquali F, Danesino C (1990) Gene dosage effect in acquired monosomy 7 : Distinct behaviour of $\beta$-glucuronidase and phosphoserine phosphatase. Genes Chromosom Cancer 1:216-220.

Mitelman F (1991) Catalog of Chromosome Aberrations in Cancer, 4th ed. New York: Wiley-Liss, pp 575-683.

Nielsen J, Wohlert M (1991) Chromosome abnormalities found among 34910 newborn children: Results from a 13-year incidence study in Arhus, Denmark. Hum Genet 87:81-83.

Oshima J, Chang-En Y, Boehnke M, Weber JL, Edelhoff S, Wagner MJ, Wells DE, Wood S, Disteche CM, Martin GM, Schellenberg GD (1994) Integrated mapping analysis of the Werner syndrome region of chromosome 8 . Genomics 23:100-113.

Palmer CG, Provisor AJ, Weaver DD, Hodes ME, Heerema N (1983) Juvenile chronic granulocytic leukemia in a patient with trisomy 8, neurofibromatosis, and prolonged Epstein-Barr virus infection. J Pediatr 102:888-892.

Procter SE, Watt JL, Lloyd DJ, Duffty P (1984) Problems of detecting mosaicism in skin. A case of trisomy 8 mosaicism illustrating the advantages of in situ tissue culture. Clin Genet 25:273277.

Riccardi VM, Humbert JR, Peakman D (1978) Acute leukemia associated with trisomy 8 mosaicism and a familial translocation 46,XY,t(7;20)(p13;p12). Am J Med Genet 2:15-21.

Robinson WP, Binkert F, Bernasconi F, Lorda-Sanchez I, Werder EA, Schinzel AA (1995) Molecular studies of chromosomal mosaicism: Relative frequency of chromosome gain or loss and possible role of cell selection. Am J Hum Genet 56:444-451.

Sadamori N, Takei H, Yao E, Nagamine M, Tomonaga Y, Tagawa M, Kusano M, Ichimaru M (1983) Erythrocyte glutathione reductase activity and acquired trisomy 8 in various hematologic disorders. Cancer Genet Cytogenet 12:255-259.

Sandberg AA (1991) Chromosome changes in human malignancy including the acute leukemias in Down syndrome. In EE McCoy, CJ Epstein (eds): Oncology and Immunology of Down Syndrome. New York: Alan R. Liss, 57-73.

Sandberg AA, Bridge JA (1994) The Cytogenetics of Bone and Soft Tissue Tumors. Austin: RG Landes, 107-109.

Schinzel A (1994) Human Cytogenetics Dacabase. Oxford, Oxford University Press.

Solomon E, Borrow J, Goddard AD (1991) Chromosome aberrations and cancer. Science 254:1153-1160. 